\title{
Incidence of Chemotherapy-Induced Nausea and Vomiting and Antiemetic Guideline Compliance in Chinese Real Clinical Setting: A Cross-Sectional Multicenter Survey
}

\section{yanxia shi ( $\sim$ shiyx@sysucc.org.cn )}

Sun Yat-Sen University Cancer Prevention and Treatment Center: Sun Yat-sen University Cancer Center

\section{Cong Xue}

Sun Yat-sen University Cancer Center

Lu Li

Sun Yat-sen University Cancer Center

\section{Qing Xia}

Shanghai Jiao Tong University School of Medicine Affiliated Renji Hospital Department of Laboratory Medicine

\section{Xin An}

Sun Yat-Sen University Cancer Prevention and Treatment Center: Sun Yat-sen University Cancer Center

\section{Haifeng Li}

Sun Yat-Sen University Cancer Prevention and Treatment Center: Sun Yat-sen University Cancer Center

\section{Lulu Zhang}

Sun Yat-Sen University Cancer Prevention and Treatment Center: Sun Yat-sen University Cancer Center

\section{Mei Hou}

Tongji Hospital of Tongji Medical College of Huazhong University of Science and Technology

\section{Xianglin Yuan}

Huazhong University of Science and Technology Tongji Medical College

\section{Xi Chen}

900th Hospital of PLA

\section{Yiping Zhang}

Zhejiang Cancer Hospital

\section{Lingdi Zhao}

Henan Provincial Tumor Hospital: Henan Cancer Hospital

\section{Yuankai Shi}

Cancer Hospital Chinese Academy of Medical Sciences

\section{Xichun Hu}

Sun Yat-sen University State Key Laboratory of Oncology in South China

\section{Shune Yang}


Xinjiang Medical University Affiliated Third Hospital: Xinjiang Medical University Affiliated Tumor Hospital

\section{Jing Cheng}

Wuhan Union Hospital

\section{Yi Luo}

Hunan Cancer Hospital

\section{Hui Liang}

Hunan Cancer Hospital

\section{Qingfeng Zou}

Guangzhou Medical University Affiliated Cancer Hospital

\section{Zhengxiang Han}

xu zhou yi xue yuan: Xuzhou Medical University

\section{Kangsheng Gu}

The First Affiliated Hospital of An Hui Medicial Hospital

\section{Zuoxing Niu}

Shan Dong Tumor Hospital

\section{Yuhuan Gao}

the Fourth Hospital of He Bei Medicial University

\section{Li Xia}

: People's Hospital of Jilin Province

\section{Runxiang Yang}

Yunnan Cancer Center: Yunnan Cancer Hospital

\section{Jianping Xiong}

The First Affiliated Hospital of Nanchang University

\section{Qing Bu}

The First Affiliated Hospital of Guangxi Medicial Universiity

\section{Xiaodong Jiang}

The First People's Hospital of Lianyungang

\section{Xiaodong Xie}

General Hospital of Northern Theatre command

\section{Tao Sun}

liao ning sheng zhong liu yi yuan: Liaoning Cancer Institute and Hospital

\section{Qingyuan Zhang}

the Affiliated Tumor Hospital of Harbin Medical University

\section{Shoucheng Ma}

Lanzhou University First Affiliated Hospital

\section{Shisheng Tan}

: Guizhou Provincial People's Hospital

\section{Miao Li}


Qing Hai Province Cancer Hospital

\section{Ningju Wang}

General Hospital of Ningxia Medical University

\section{Xiumei Wang}

Inner Mongolia Cancer Hospital

\section{Yi Ba}

Tianjin Medical University Cancer Institute and Hospital: Tianjin Tumor Hospital

\section{Jifeng Feng}

Jiangsu Cancer Hospital

\section{Wenqi Jiang}

Sun Yat-Sen University Cancer Prevention and Treatment Center: Sun Yat-sen University Cancer Center

\section{Research Article}

Keywords: chemotherapy-induced nausea and vomiting (CINV), antiemetics, guideline compliance, real clinical setting

Posted Date: November 1st, 2021

DOI: https://doi.org/10.21203/rs.3.rs-457938/v2

License: (c) (i) This work is licensed under a Creative Commons Attribution 4.0 International License. Read Full License 


\section{Abstract \\ Background}

This survey aims to investigate the incidence of chemotherapy-induced nausea and vomiting (CINV) in Chinese real clinical setting and evaluate the effect of guideline-consistent CINV prophylaxis (GCCP) and guideline-inconsistent CINV prophylaxis (GICP) on incidence of complete response (CR) of CINV.

\section{Materials and Methods}

A cross-sectional nationwide multicenter study assessing the guideline consistency and CINV incidence of patients was conducted at a total of 32 large medical centers from 26 provinces across the west,east $\llbracket$ northeast and middle part of China between April and May 2021.

\section{Result}

Data for 2964 patients were analyzed. Patients treated with moderately emetogenic chemotherapy (MEC) were more prone to experience CINV during the acute phase compared to those receiving highly emetogenic chemotherapy (HEC); patients receiving low or minimally emetogenic chemotherapy $(\mathrm{L} / \mathrm{mEC})$ were least likely to experience CINV during the overall phase among the whole study population. The prevalence of GCCP was $29.2 \%$ in the whole study population, and $13.6 \%, 35.7 \%$ and $45.1 \%$ for the patients receiving $\mathrm{HEC}, \mathrm{MEC}$ and $\mathrm{L} / \mathrm{mEC}$, respectively. For patients receiving $\mathrm{HEC}$ and $\mathrm{MEC}, \mathrm{GCCP}$ increased incidence of $\mathrm{CR}$ during both delayed and overall phases. For those receiving $\mathrm{L} / \mathrm{mEC}$ and GICP, incidence of $C R$ was not higher than that of patients receiving $L / m E C$ and GCCP.

\section{Conclusion}

This study revealed Chinese CINV status, the prevalence of GCCP in the real clinical setting and the association between GCCP and CR rate for the first time. The findings indicate that prescribing antiemetics in compliance with guidelines for all patients receiving chemotherapy is strongly suggested.

\section{Implications For Practice}

The prevalence of GCCP in the real clinical setting was unknown in China, despite international antiemetic guidelines have been issued and regularly update. Findings in this study revealed that GCCP prevalence needs improvement and GCCP has a positive effect on the incidence of CR. Therefore, it is highly recommended that oncologists prescribe guideline-consistent antiemetics for cancer patients receiving chemotherapy in the real clinical setting.

\section{Introduction}


Chemotherapy-induced nausea and vomiting (CINV) is considered as a common, debilitating reaction to chemotherapeutic agents[1]. The incidence of CINV has been well controlled as the development of effective antiemetics[2]. Although effective antiemetics and consensus guidelines for CINV are available nowadays, CINV still deteriorate patients' quality of life and in the worst case even lead to discontinuation of chemotherapy, compromised effectiveness of chemotherapy and more costs on outpatient visits and hospitalization[3].

According to international guideline, chemotherapy regimens are categorized into four classes based on the antiemetic with the highest emetogenicity in the regimen[4], which are highly emetogenic chemotherapy (HEC), moderately emetogenic chemotherapy (MEC), low emetogenic chemotherapy (LEC), and minimally emetogenic chemotherapy, respectively. CINV could occur in a biphasic mode consisting of acute $(\leq 24 \mathrm{~h}$ from administration of chemotherapy,day 1$)$ phase and delayed $\nabla 24 \mathrm{~h}$ from administration of chemotherapy, days 2-5区 phase. Previous studies reported that CINV was more prone to occur in the delayed phase as compared with the acute phase[5].

Guideline consistency in practice is variable across different countries, Studies conducted in the United states and Europe have reported the unsatisfactory incidence of guideline adherence which were $57.3 \%$ and $29 \%$, respectively, and the findings in these two studies indicated that compliance with antiemetic guidelines has a positive effect on control of CINV during the overall phase (acute phase plus delayed phase) for patients administered HEC or MEC[6, 7]. Thus the primary objective of this survey was to analyze the impact of guideline-consistent CINV prophylaxis (GCCP) and guideline-inconsistent CINV prophylaxis (GICP) on incidence of complete response (CR) in China. Besides, a number of clinical trials reported that use of newly modern antiemetics could increase rate of complete response (CR), such as palonosetron (PALO) and aprepitant [8, 9]. Considering few studies about the effectiveness of 5hydroxytryptamine-3 receptor antagonist (5HT3-RA) or aprepitant in combined regimens in the real clinical setting, investigating their effectiveness in combined regimens in Chinese clinical practice was also conducted.

\section{Materials And Methods}

This was a cross-sectional nationwide multicenter survey conducted at 32 large medical centers including cancer centers and general hospitals from 26 provinces across the west,east $\square$ northeast and middle part of China between April and May 2021. To keep balance, the number of patients at each study site was requested to range from 30 to 120 . All methods were carried out in accordance with relevant guidelines and regulations. All experimental protocols were approved by Ethics Committee of Sun Yat-sen University Cancer Center. Informed consent was obtained from all subjects and all subjects are above 18, and the ethical approval was provided in supplement 2 .

\section{Patients}


Patients who were $\geq 18$ years old and had already received HEC, MEC, low or minimally emetogenic chemotherapy $(\mathrm{L} / \mathrm{mEC})$ were eligible for the study. Patients were excluded if they had been received opioids or other drugs that interfere with the metabolism of antiemetics during chemotherapy treatment, and those who cannot express objective discomfort, cannot take food because of digestive tract obstruction, with brain metastases and concurrent radiation therapy were also excluded.

\section{Questionnaire design and outcomes}

The questionnaire included six sections as follows: demographic information, patients' recall about control of CINV after the most recent chemotherapy, antiemetic regimens, adverse effects of antiemetics, treatment adherence and medical costs. Oncologists filled out the questionnaire via asking patients predesigned questions (Questionnaire is presented in supplemental 1).

Analyzing the data from questionnaire led to following outcomes: demographics, use of antiemetic medications for CINV, incidence of CINV, the effect of GCCP and GICP on incidence of complete response (CR) and prognostic factors for the overall CR. CR referred to no vomiting and no rescue therapy. This study analyzed CR during the acute, delayed and overall phases, respectively. The Emetogenicity of chemotherapy regimen was categorized into four classes,and the definition of GCCP in this study was based on the NCCN Antiemesis Guidelines Version 1.2019 [4] (supplemental Table 1). In our study, LEC and minimally emetogenic chemotherapy were analyzed as a group named $\mathrm{L} / \mathrm{mEC}$. For patients receiving LEC, GCCP was defined as use of single agent as follows: dexamethasone, metoclopramide, prochlorperazine or 5HT3-RA; using more than one antiemetic for patients receiving LEC was regarded as GICP; for patients receiving minimally emetogenic chemotherapy, no routine prophylaxis was defined as GCCP.

\section{Statistical Analyses}

Demographics and clinical characteristics of patients were compared among HEC,MEC $\square \mathrm{L} / \mathrm{mEC}$ groups using Chi-square test for categorical variables and Kruskal-Wallis test for age; post-hoc analysis was used to compare difference in distribution for gender and use of antiemetic therapy. Chi-square test was used to compare the incidence of CR between GCCP and GICP. Prognostic factors for CR during the overall period were identified via univariate and multivariate logistic regression analysis. The independent factors included in the analysis are as follows: gender, age, number of prophylactic antiemetics, use of 5HT3-RA, emetogenic risk level of chemotherapy, use of aprepitant, history of anticipatory nausea and vomiting (ANV), education level, degree of knowing illness and guideline compliance[10-12].Two-sided $P$ values $<0.05$ were accepted as statistically significant. STATA software, version 15.0 was used for all statistical analyses.

\section{Ethical Considerations}

This cross-sectional multicenter survey was initiated by the Clinical Chemotherapy Committee of Chinese Anti Cancer Association (CACA) and the contents of questionnaire enshrine ethical considerations, without any invasion of privacy. All methods were carried out in accordance with relevant guidelines and 
regulations. All experimental protocols were approved by Ethics Committee of Sun Yat-sen University Cancer Center. Informed consent was obtained from all subjects and all subjects are above 18 , and the ethical approval was provided in supplement 2.

\section{Result}

\section{Patients}

Of 3000 patients enrolled, as data for chemotherapy regimen were missing for 36 patients, data for 2964 patients treated with HEC, MEC or L/mEC were analyzed. Table 1 summarizes the characteristics of the 2964 patients (1184 treated with HEC, 1037 treated with MEC and 743 treated with L/mEC; 1516 men and 1448 women; 1514 and 1450 patients from cancer center and general hospital, respectively). The median ages in HEC, MEC and L/MEC were 56,56 and 57 years old, respectively. The 3 groups (HEC, MEC, L/mEC) of patients shared an unsimilar distribution of gender, prevalence of antiemetic usage, degree of knowing illness and percentages of cancer type $(P<0.01)$. General hospital and cancer center showed a similar distribution among 3 groups $(P=0.179)$. Greater proportions of male patients were in HEC and MEC cohort compared with L/MEC cohort $(P<0.001)$. A lower usage rate of antiemetics in MEC and L/mEC compared to HEC cohort $(P<0.001)$. 
Table 1

Demographics and clinical characteristics of included patients $(\mathrm{N}=2,964)$

\begin{tabular}{|c|c|c|c|c|c|}
\hline & $\begin{array}{l}N= \\
2,964\end{array}$ & $\begin{array}{l}\text { HEC } \\
(N=1,184)(\%)\end{array}$ & $\begin{array}{l}\text { MEC } \\
(N=1,037)(\%)\end{array}$ & $\begin{array}{l}\mathrm{L} / \mathrm{mEC} \\
(\mathrm{N}=743)(\%)\end{array}$ & $p^{\mathrm{a}}$ \\
\hline $\begin{array}{l}\text { Age, years, median } \\
\text { (IQR) }\end{array}$ & 2,945 & $\begin{array}{l}56(47,64)(n= \\
1,179)\end{array}$ & $\begin{array}{l}56(49,65)(n= \\
1,030)\end{array}$ & $\begin{array}{l}57(49,66)(n= \\
736)\end{array}$ & 0.011 \\
\hline Gender & & & & & $<0.001$ \\
\hline Male & 1,516 & $616(52.0)$ & 587 (56.6) & $313(42.1)$ & \\
\hline Female & 1,448 & $568(48.0)$ & $450(43.4)$ & 430 (57.9) & \\
\hline Type of hospital & & & & & 0.179 \\
\hline General hospital & 1,450 & 559 (47.2) & $508(49.0)$ & 383 (51.5) & \\
\hline Cancer center & 1,514 & $625(52.8)$ & $529(51.0)$ & $360(48.5)$ & \\
\hline $\begin{array}{l}\text { Use of antiemetic } \\
\text { therapy }\end{array}$ & & & & & $<.001$ \\
\hline No & 24 & $0(0.0)$ & $10(1.0)$ & $14(1.9)$ & \\
\hline Yes & 2,940 & $1184(100.0)$ & 1027 (99.0) & 729 (98.1) & \\
\hline ANV & & & & & 0.138 \\
\hline No & 2,609 & 1,059 (89.4) & $900(86.8)$ & $650(87.5)$ & \\
\hline Yes & 355 & $125(10.6)$ & 137 (13.2) & $93(12.5)$ & \\
\hline Education & & & & & 0.086 \\
\hline Low & 2,241 & 906 (81.1) & 764 (77.8) & 571 (81.6) & \\
\hline High & 558 & 211 (18.9) & 218 (22.2) & 129 (18.4) & \\
\hline $\begin{array}{l}\text { Degree of knowing } \\
\text { illness }\end{array}$ & & & & & 0.003 \\
\hline Not at all & 176 & $68(5.8)$ & $66(6.5)$ & $42(5.8)$ & \\
\hline A little & 1,740 & 733 (62.4) & $615(60.8)$ & $392(54.1)$ & \\
\hline Completely known & 994 & $373(31.8)$ & $330(32.6)$ & $291(40.1)$ & \\
\hline
\end{tabular}

Values represent number (\%) of patients unless otherwise noted.

${ }^{\text {a}}$ Chi-square test for categorical data and Kruskal-Wallis test for age (skewed distribution).

Abbreviation: HEC, highly emetogenic chemotherapy;MEC, moderately emetogenic chemotherapy; $\mathrm{L} / \mathrm{MEC}$, low/minimally emetogenic chemotherapy; IQR,Interquartile range; ANV, anticipatory nausea and vomiting. 


\begin{tabular}{|c|c|c|c|c|c|}
\hline & $\begin{array}{l}N= \\
2,964\end{array}$ & $\begin{array}{l}\text { HEC } \\
(N=1,184)(\%)\end{array}$ & $\begin{array}{l}\text { MEC } \\
(N=1,037)(\%)\end{array}$ & $\begin{array}{l}\mathrm{L} / \mathrm{mEC} \\
(\mathrm{N}=743)(\%)\end{array}$ & $p^{a}$ \\
\hline Type of cancer & & & & & $<.001$ \\
\hline Lung cancer & 788 & $485(61.6)$ & $90(11.4)$ & $213(27)$ & \\
\hline Breast cancer & 525 & $230(43.8)$ & 68(13.0) & $227(43.2)$ & \\
\hline $\begin{array}{l}\text { Head and neck } \\
\text { cancer }\end{array}$ & 84 & $44(52.4)$ & $26(30.9)$ & 14(16.7) & \\
\hline $\begin{array}{l}\text { Digestive system } \\
\text { cancer }\end{array}$ & 994 & $89(9.0)$ & 695(69.9) & $210(21.1)$ & \\
\hline $\begin{array}{l}\text { Gynecological } \\
\text { cancer }\end{array}$ & 102 & $54(52.9)$ & $33(32.4)$ & 15(14.7) & \\
\hline $\begin{array}{l}\text { Hematological } \\
\text { cancer }\end{array}$ & 244 & 157(64.3) & $69(28.3)$ & 18(7.4) & \\
\hline Urogenital cancer & 80 & $48(60.0)$ & $12(15.0)$ & $20(25.0)$ & \\
\hline Sarcoma & 19 & 15(78.9) & $1(5.3)$ & $3(15.8)$ & \\
\hline Melanoma & 11 & $4(36.4)$ & $4(36.4)$ & $3(27.2)$ & \\
\hline Others & 117 & $58(49.6)$ & $39(33.3)$ & $20(17.1)$ & \\
\hline \multicolumn{6}{|c|}{ Values represent number (\% ) of patients unless otherwise noted. } \\
\hline \multicolumn{6}{|c|}{${ }^{\text {a}}$ Chi-square test for categorical data and Kruskal-Wallis test for age (skewed distribution). } \\
\hline
\end{tabular}

\section{Antiemetic treatment and CINV status in the real world}

65.69\% (1947/2964) were given a combined regimen while 33.50\% (993/2964) were given a single agent. Among patients who received single agent, most of them $(97.99 \%, 973 / 993)$ were prescribed 5HT3-RA. 5HT3-RA and corticosteroid was the most common prescribed regimen among combined regimens $(41.50 \%, 808 / 1947)$ (supplemental Table 2). 5HT3-RA nearly became the basis of an antiemetic regimen as 2876 out of 2964 patients had 5HT3-RA in their prophylaxis antiemetic regimens. PALO was the most commonly prescribed 5HT3-RA $(n=1068 ; 37.13 \%)$, followed by Tropisetron $(n=817 ; 28.41 \%)$, and only $2.68 \%(n=77)$ were administered Azasetron which was the least used 5HT3-RA.

CR outcomes of two specific antiemetic regimens for HEC and MEC were describes respectively in supplemental Table 3. The patients treated with HEC and aprepitant-containing triple antiemetics (5HT3$\mathrm{RA}+$ dexamethasone + aprepitant) had statistically higher CR rates compared with patients treated with 
dual antiemetics (5HT3-RA + dexamethasone) during the acute phase and overall phase; but for the patients treated with MEC, the CR rate had no significant difference between above-mentioned dual antiemetics and triple antiemetics. Among the patients after HEC and triple antiemetics(5HT3-RA + dexamethasone + aprepitant), the CR rates between patients treated with PALO and patients treated with 1st generation 5HT3-RA did not show a statistically significant difference. Regarding the patients after MEC treated with dual antiemetics (5HT3-RA + dexamethasone) or triple antiemetics (5HT3-RA + dexamethasone + aprepitant), the CR rate in patients prescribed PALO was not statistically different from that in patients prescribed 1 st generation 5HT3-RA.

During overall, acute and delayed phases, patients were more likely to experience nausea compared with vomiting (Fig. 1). During the overall phase, $33.4 \%$ of the whole study population experienced nausea while $9.7 \%$ experienced vomiting; patients administered MEC were most likely to experience nausea (38.3\%). Patients receiving MEC also had the highest incidence of nausea and vomiting in the acute phase $(53.2 \%$ and $22.9 \%$, respectively). In the delayed phase, the incidence of vomiting in patients receiving HEC was highest (19.6\%) while the incidence of nausea in patients receiving MEC was higher than that in patients receiving HEC (47.9\% vs $45.7 \%)$. The incidence of acute nausea in patients receiving MEC and L/mEC was higher than that of delayed nausea (MEC: $53.2 \%$ vs $47.9 \%$; L/mEC: $41.3 \%$ vs $38 \%$ ). The incidence of acute vomiting in patients receiving HEC was slightly higher than the incidence of delayed vomiting (20.7\% vs $19.6 \%$ ), and for patients receiving MEC and $\mathrm{L} / \mathrm{mEC}$, they were both more likely to vomit in the acute phase compared to the delayed phase $(22.9 \%$ vs $17 \%, 18.4 \%$ vs $14.5 \%$, respectively).

\section{GCCP vs GICP}

Between the patients treated in cancer center and those treated in general hospital, the guideline compliance showed no statistically significant difference ( $51.2 \%$ vs $48.8 \%, P=0.95)$. The prevalence of GCCP was $29.2 \%$ (866/2964) in the whole study population, and $13.6 \%(161 / 1184), 35.7 \%(370 / 1037)$ and $45.1 \%$ (335/743) for the patients receiving HEC, MEC and L/mEC, respectively. As shown in Fig. 2, for the whole study population, higher incidence of CR in patients treated with GCCP was observed during overall, acute and delayed phases ( $P=0.023$ for acute $\mathrm{CR} ; P<0.001$ for both delayed and overall $\mathrm{CR}$ ). GCCP could significantly increase the incidence of CR in the patients treated with HEC $(P=0.007$ for the delayed $\mathrm{CR} ; P<0.001$ for the acute and overall $\mathrm{CR}$ ). Regarding the patients treated with MEC, the incidence of acute $\mathrm{CR}$ in patients receiving GCCP was not statistically higher than that in the patients receiving GICP $(P=0.311)$, but $C R$ rates were both statistically higher in the patients treated with GCCP than that in the patients treated with GICP for both delayed and overall phases $(P=0.042, P=0.007$, respectively). For the patients treated with $\mathrm{L} / \mathrm{mEC}$, no statistically significant difference in $\mathrm{CR}$ rates between GCCP and GICP was observed during the acute, delayed and overall phases.

\section{Univariate and Multivariate analysis of prognostic factors for overall CR}


Univariate and multivariate logistic regression analyses were performed to identify several statistically significant predictors for overall CR (Table 2). The univariate analysis showed that male sex, older age, two antiemetics versus single antiemetic, three antiemetics versus single antiemetic, L/mEC versus HEC, treatment with aprepitant, without ANV and GCCP were all statistically significant prognostic factors for overall CR. The multivariate analysis revealed that male sex (odds ratio [OR] for female sex, $0.542 ; 95 \%$ confidence interval $[\mathrm{Cl}] 0.453-0.649 ; \mathrm{P}<0.001)$, treatment with aprepitant $(\mathrm{OR}, 2.102 ; 95 \% \mathrm{Cl}, 1.086-$ $4.065 \rrbracket P=0.027$ ) $\llbracket$ without ANV (OR for with ANV, $0.277 ; 95 \% \mathrm{Cl}, 0.217-0.353 ; P<0.001$ ), two antiemetics versus single antiemetic (OR, 1.273;95\% $\mathrm{Cl}, 1.054-1.537 ; \mathrm{P}=0.012), \mathrm{L} / \mathrm{MEC}$ versus $\mathrm{HEC}(\mathrm{OR}, 1.653 ; 95 \% \mathrm{Cl}$, $1.309-2.087 ; P<0.001)$ and $G C C P(O R, 1.369 ; 95 \% \mathrm{Cl}, 1.084-1.729 ; P=0.008)$ were statistically significant independent predictors for overall $\mathrm{CR}(P<0.05)$. 
Table 2

Univariate and multivariate logistic regression model for overall $C R$

\begin{tabular}{|c|c|c|c|c|}
\hline & $\begin{array}{l}\text { Univariate } \\
\text { analysis }\end{array}$ & & $\begin{array}{l}\text { Multivariate } \\
\text { analysis }\end{array}$ & \\
\hline Factor & OR $(95 \% \mathrm{Cl})$ & $P$ & OR $(95 \% \mathrm{Cl})$ & $P$ \\
\hline Gender: female vs. male & $\begin{array}{l}0.537(0.456- \\
0.631)\end{array}$ & $<.001$ & $\begin{array}{l}0.541(0.452- \\
0.648)\end{array}$ & $\begin{array}{l}< \\
0.001\end{array}$ \\
\hline Age (continuous variable) & $\begin{array}{l}1.016(1.010- \\
1.023)\end{array}$ & $<0.001$ & $1.007(1.000-1.015)$ & 0.056 \\
\hline $\begin{array}{l}\text { Number of prophylactic } \\
\text { antiemetics } \\
\text { one }\end{array}$ & Reference & & & \\
\hline Two & $\begin{array}{l}1.283(1.081- \\
1.523)\end{array}$ & 0.004 & $\begin{array}{l}1.305(1.080- \\
1.578)\end{array}$ & 0.006 \\
\hline Three & $\begin{array}{l}2.295(1.625- \\
3.240)\end{array}$ & $<0.001$ & $\begin{array}{l}0.983(0.451- \\
2.141)\end{array}$ & 0.965 \\
\hline Four & $\begin{array}{l}1.158(0.698- \\
1.920)\end{array}$ & 0.570 & $\begin{array}{l}0.620(0.264- \\
1.455)\end{array}$ & 0.272 \\
\hline 5HT3-RA: Yes vs. No & $\begin{array}{l}0.729(0.440- \\
1.208)\end{array}$ & 0.219 & $\begin{array}{l}0.732(0.388- \\
1.378)\end{array}$ & 0.333 \\
\hline Chemotherapy & Reference & & & \\
\hline \multicolumn{5}{|l|}{ HEC } \\
\hline MEC & $\begin{array}{l}1.024(0.853- \\
1.229)\end{array}$ & 0.802 & $\begin{array}{l}1.085(0.880- \\
1.339)\end{array}$ & 0.445 \\
\hline $\mathrm{L} / \mathrm{mEC}$ & $\begin{array}{l}1.259(1.024- \\
1.549)\end{array}$ & 0.029 & $\begin{array}{l}1.462(1.142- \\
1.873)\end{array}$ & 0.003 \\
\hline Aprepitant: Yes vs. No & $\begin{array}{l}1.712(1.327- \\
2.209)\end{array}$ & $<0.001$ & $\begin{array}{l}2.188(1.131- \\
4.234)\end{array}$ & 0.020 \\
\hline ANV: Yes vs. No & $\begin{array}{l}0.292(0.233- \\
0.367)\end{array}$ & $<0.001$ & $\begin{array}{l}0.275(0.216- \\
0.351)\end{array}$ & $\begin{array}{l}< \\
0.001\end{array}$ \\
\hline Education: High vs. low & $\begin{array}{l}0.952(0.777- \\
1.167)\end{array}$ & 0.637 & $\begin{array}{l}1.037(0.826- \\
1.301)\end{array}$ & 0.755 \\
\hline
\end{tabular}

Abbreviation: $\mathrm{Cl}$, confidence interval; ANV, Anticipatory nausea and vomiting; HEC,highly emetogenic chemotherapy;MEC, moderately emetogenic chemotherapy; L/MEC, low/minimally emetogenic chemotherapy; OR】odds ratio; GCCP』guideline-consistent CINV (chemotherapy induced nausea and vomiting) prophylaxis $\square \mathrm{GICP} \square$ guideline-inconsistent $\mathrm{CINV} \square$ chemotherapy induced nausea and vomiting \prophylaxis. 


\section{Univariate \\ analysis}

\section{Multivariate analysis}

Degree of knowing illness

Not at all Reference

\begin{tabular}{|c|c|c|c|c|}
\hline A little & $\begin{array}{l}1.120(0.797- \\
1.575)\end{array}$ & 0.514 & $\begin{array}{l}0.957(0.657- \\
1.395)\end{array}$ & 0.821 \\
\hline Completely known & $\begin{array}{l}1.013(0.713- \\
1.440)\end{array}$ & 0.942 & $\begin{array}{l}0.870(0.588- \\
1.287)\end{array}$ & 0.485 \\
\hline GCCP vs. GICP & $\begin{array}{l}1.472(1.226- \\
1.767)\end{array}$ & $<.001$ & $\begin{array}{l}1.369(1.084- \\
1.729)\end{array}$ & 0.008 \\
\hline
\end{tabular}

Abbreviation: $\mathrm{Cl}$, confidence interval; $\mathrm{ANV}$, Anticipatory nausea and vomiting; $\mathrm{HEC}$,highly emetogenic chemotherapy;MEC, moderately emetogenic chemotherapy; L/MEC, low/minimally emetogenic chemotherapy; OR】odds ratio; GCCP』guideline-consistent CINV (chemotherapy induced nausea and vomiting) prophylaxis $\square \mathrm{GICP} \square$ guideline-inconsistent $\mathrm{CINV} \llbracket$ chemotherapy induced nausea and vomiting 『prophylaxis.

\section{Discussion}

For the first time this study revealed Chinese CINV status, the prevalence of GCCP in the real clinical setting and the association between GCCP and CR rate. The findings in this study indicate that CR rate in the patients treated with GCCP beyond that seen with patients treated with GICP, thus standardizing the use of antiemetics in real clinical setting is of great significance. The strengths of this study lie in many aspects. It consisted of a total of 32 large medical centers and 2964 patients from 26 provinces of China. Compared with the previous western studies[6,7], the bigger sample size in this study provided a more robust evaluation of the benefits of GCCP. More notably, the incidence of CR after patients receiving $\mathrm{L} / \mathrm{mEC}$ was compared between GCCP and GICP for the first time[13], as patients treated with L/mEC were not enrolled in previous western studies.

This study illustrated that current antiemetic regimens in compliance with NCCN guideline effectively control chemotherapy-induced emesis in patients receiving HEC and MEC. In an American study (INSPIRE), Gilmore et al. found, among patients treated with HEC, higher rate of no CINV in GCCP group compared with GICP group during both acute and delayed phases $(49.2 \%$ vs $37.8 \%, P=0.024)$ [7]. For the patients treated with MEC in this study, although the positive effect of GCCP on acute CR rate was not statistically significant, INSPIRE study reported that rate of no emesis in patients treated with MEC and GCCP was statistically higher than that in GICP group ( $91.6 \%$ vs $81.2 \%, P=0.020$ ) during the overall phase[7]. Aapro et al. in Pan European Emesis Registry (PEER) study indicated that in Europe, the incidence of CR in GCCP was significantly higher than it was in GICP during the acute, delayed phases and overall period (OR 1.43, $P=0.027)[6]$. So findings in this study support prescribing antiemetics in compliance with guidelines in the real clinical setting for patients receiving HEC, MEC as GCCP could significantly increase the incidence of CR for patients receiving HEC and MEC. Besides, for the patients 
receiving $\mathrm{L} / \mathrm{mEC}$, no significant difference in $\mathrm{CR}$ rate between GCCP and GICP was observed, indicating that excessive treatment (more than one antiemetic) for patients receiving LEC and prescribing antiemetics for patients receiving minimally emetogenic chemotherapy could not increase the incidence of CR.

In this study, only $13.6 \%$ of patients prescribed HEC and $35.7 \%$ of patients prescribed MEC complied the NCCN 2019 guidelines, respectively, which were comparable with the results reported in the conducted by Aapro et al. (11\%, 39\%, respectively). However, this was not a satisfactory result when compared with the GCCP prevalence in a Japanese study, in which Hirotoshi et al. reported $96 \%$ of patients receiving carboplatin-based chemotherapy (HEC and MEC were both included) were in compliance with the antiemesis guidelines based on JSCO 2010 guidelines[14]. Except the fact of using different guidelines, Japan enacted the first cancer control law in 2006, which confirmed that political policies could influence compliance with guidelines[14]. Economic burden is another notable issue. The cost-effectiveness of using aprepitant was reported quite good in Germany, the United States and the United Kingdom[15]. But it has not been involved into Chinese health insurance, which might be responsible for the insufficient use of aprepitant in patients receiving HEC (only 255 out of 1184 patients receiving HEC were administered aprepitant in this survey), hence improving health care policy might be a practical measure to increase guideline consistency and reduce the burden of CINV.

The incidence of nausea during overall, acute and delayed phases was more frequent than vomiting, which was consistent with previous studies[16, 17]. Ng, T. L et al. reported that patients ranked nausea more of a problem than vomiting[18]. The incidence of nausea during the overall phase was approximately 3.5-fold higher than that in the vomiting. This implies the control of nausea in real clinical setting was much worse compared to the control of vomiting. For the overall study population, data generated in this study found that the incidence of acute CINV was higher than the incidence of delayed CINV, unlike results reported by previous studies indicating that incidence of delayed CINV was higher[16, 20].This might because those previous studies analyzed first cycle of chemotherapy among chemotherapy-naïve patients [21]. while our study, as a cross-sectional study, referred to patients' most recent treatment cycle consisting of diverse chemotherapy regimens, confounding factors were inevitable. Vidall et al found the same pattern of CINV incidence as our study which might because they also referred to patients' most recent cycle of chemotherapy[22]. The consistency might imply the incidence of CINV in the acute phase was indeed higher compared with delayed phase in real clinical setting. Unexpectedly, higher percentage of patients receiving MEC experienced nausea compared to patients receiving HEC, although the prevalence of GCCP in MEC was higher than that in HEC. This could be attributed to the insufficient education and contacts between oncologists and patients receiving MEC, unlike the patients receiving HEC, who were more prone to be educated about the prophylaxis of CINV.

5HT3-RA was the basis of an antiemetic regimen as $97.03 \%$ of patients had 5HT3-RA in their prophylaxis antiemetic regimens. A phase III clinical study reported that among different types of 5HT3-RA, PALO as the most commonly prescribed, its complete response (CR) rates for CINV were significantly higher compared with 1st generation 5HT3-RA in the delayed and overall phases[8]. Two large phase III trials 
involving patients treated with MEC showed higher rate of preventing vomiting with PALO than with ondansetron or dolasetron[23, 24]. But in our study, regarding MEC, among patients treated with guideline-consistent dual antiemetics (5HT3-RA + dexamethasone) or triple antiemetics (5HT3-RA + dexamethasone + aprepitant), PALO did not show a higher CR rate when compared with the 1st generation 5HT3-RA. The reason might because in previous clinical trials patients received single agent, while in our clinical setting, most patients received combined antiemetic regimens. Therefore, in terms of combined antiemetic regimens in the real clinical setting, selection between PALO and 1st generation 5HT3-RA needs further verification.

Regarding the patients receiving HEC, the addition of aprepitant into dual antiemetics is of great significance as triple antiemetics (5HT3-RA + dexamethasone + aprepitant) had higher CR rates in acute and overall phases compared with dual antiemetics (5HT3-RA + dexamethasone). This result was comparable with the study by Navari et al., in which they found addition of aprepitant to a standard regimen of 5HT3-RA and dexamethasone in patients receiving HEC improves the CR rate of acute CINV[9]. Two trials involving patients receiving HEC reported significantly higher efficacy in the control of emesis with the addition of aprepitant to ondansetron plus dexamethasone than with ondansetron plus dexamethasone alone[25, 26], therefore, the efficacy and effectiveness of aprepitant were both confirmed via previous RCTs and this cross-sectional study in the real clinical setting.

This study identified several independent predictors for overall CR in accordance with previous studiesmale, use of aprepitant and no ANV (anticipatory nausea and vomiting). Besides, our results indicated that GCCP, use of aprepitant,two antiemetics (versus single agent) and L/mEC (versus HEC) were the protective factors for overall CR. Differing from the widely accepted view, this study did not identify older age as the protective factor [21]. The reason might be attributed to the median age in our study was 56 years old, which led to an age strata bias.

The main limitation of this study was the data collection was based on patients' recall about the most recent treatment, which led to more missing records compared to the records from patients' daily diary. Another limitation was the results in this study may not be generalized to suburban areas in China. So above-mentioned weakness could have introduced recall bias and selection bias. Besides, most patients participated in this study were not naïve to chemotherapy, as a result, there might be more confounding factors leading to CINV, such as prior experience of poorly controlled emesis, anxiety about chemotherapy. Despite above limitations, the results in this survey supported previous studies and further illustrated the positive effect of antiemetic guideline on controlling CINV.

\section{Conclusion}

This nationwide study reported Chinese CINV status, the prevalence of GCCP and the association between GCCP and CR rate in the real clinical setting for the first time. The findings in this study indicated that prevalence of GCCP still have much room for improvement and the incidence of CR in GCCP was higher than that in GICP, thus it might be the best choice for patients to comply with GCCP in the real 
clinical setting. As such, prescribing antiemetics in compliance with guidelines for all patients receiving chemotherapy is strongly suggested.

\section{Declarations}

\section{Ethics approval and consent to participate}

All methods in this study were carried out in accordance with relevant guidelines and regulations. All experimental protocols were approved by Ethics Committee of Sun Yat-sen University Cancer Center. Informed consent was obtained from all subjects and all subjects are above 18, and the ethical approval was provided in supplement 2 .

\section{Consent for publication}

All authors have approved the manuscript and agreed with publication.

\section{Availability of data and materials}

The datasets used and/or analysed during the current study available from the corresponding author on reasonable request.

\section{Competing interests}

The authors have no conflicts of interest to declare in this work.

\section{Funding}

This work was supported by the National Natural Science Foundation of China [81773279 to Dr. Yanxia Shi], Science and Technology Planning Project of Guangdong Province [2016A050502015, 2013B021800062 and 2012B061700082 to Dr. Yanxia Shi] and Young Teacher Foundation of Sun Yatsen University [17ykzd33 to Dr. Yanxia Shi].

\section{Authors' contributions}

Conception/design: Cong Xue, Lu Li, Qing Xia, Yanxia Shi, Wenqi Jiang, Jifeng Feng

Collection and/or assembly of data: Xin An, Haifeng Li, Lulu Zhang, Mei Hou, Xianglin Yuan, Xi Chen,Yi Ba, Yiping Zhang, Lingdi Zhao, Yuankai Shi, Xichun Hu, Shune Yang, Jing Cheng,Yi Luo, Hui Liang, Qingfeng Zou, Zhengxiang Han,Kangsheng Gu, Zuoxing Niu, Yuhuan Gao, Li Xia, Runxiang Yang, Jianping Xiong, Qing Bu, Xiaodong Jiang, Xiaodong Xie, Tao Sun, Qingyuan Zhang, Shoucheng Ma, Shisheng Tan, Miao Li, Ningju Wang, Xiumei Wang

Data analysis and interpretation: Cong Xue, Lu Li, Qing Xia, Yanxia Shi 
Manuscript writing: Cong Xue, Lu Li, Qing Xia

Final approval of manuscript: All authors

\section{Acknowledgments}

This work was supported by the National Natural Science Foundation of China [81773279 to Dr. Yanxia Shi], Science and Technology Planning Project of Guangdong Province [2016A050502015, 2013B021800062 and 2012B061700082 to Dr. Yanxia Shi] and Young Teacher Foundation of Sun Yatsen University [17ykzd33 to Dr. Yanxia Shi]. We thank the Clinical Chemotherapy Committee of Chinese Anti Cancer Association (CACA) and the Chinese Journal of the Frontiers of Medical Science (Electronic Version) Editorial Department of People's Medical publishing house for initiating this survey. We also thank all study participants and investigators for their cooperation and participation.

\section{References}

1. Naito $Y$, Kai Y, Ishikawa T et al (2020) Chemotherapy-induced nausea and vomiting in patients with breast cancer: a prospective cohort study. Breast Cancer 27:122-128

2. Navari RM, Longo DL, Aapro M (2016) Antiemetic Prophylaxis for Chemotherapy-Induced Nausea and Vomiting. N Engl J Med 374:1356-1367

3. Craver C, Gayle J, Balu S, Buchner D (2011) Clinical and economic burden of chemotherapy-induced nausea and vomiting among patients with cancer in a hospital outpatient setting in the United States. J Med Econ 14:87-98

4. National Comprehensive Cancer Network. NCCN Clinical Practice Guidelines in Oncology: Antiemesis. Version 1.2019[OL].(2019-02-28)[2019-8-30]. http://www.nccn.org/professionals/physician_gls/pdf/antiemesis.pdf. In

5. Fernandez-Ortega P, Caloto MT, Chirveches E et al (2012) Chemotherapy-induced nausea and vomiting in clinical practice: impact on patients' quality of life. Support Care Cancer 20:3141-3148

6. Aapro M, Molassiotis A, Dicato M et al (2012) The effect of guideline-consistent antiemetic therapy on chemotherapy-induced nausea and vomiting (CINV): the Pan European Emesis Registry (PEER). Ann Oncol 23:1986-1992

7. Gilmore JW, Peacock NW, Gu A et al (2014) Antiemetic guideline consistency and incidence of chemotherapy-induced nausea and vomiting in US community oncology practice: INSPIRE Study. J Oncol Pract 10:68-74

8. Schwartzberg L, Barbour SY, Morrow GR et al (2014) Pooled analysis of phase III clinical studies of palonosetron versus ondansetron, dolasetron, and granisetron in the prevention of chemotherapyinduced nausea and vomiting (CINV). Support Care Cancer 22:469-477

9. Navari RM (2003) Pathogenesis-based treatment of chemotherapy-induced nausea and vomitingtwo new agents. J Support Oncol 1:89-103 
10. Molassiotis A, Aapro M, Dicato $M$ et al. Evaluation of risk factors predicting chemotherapy-related nausea and vomiting: results from a European prospective observational study. J Pain Symptom Manage 2014; 47: 839-848 e834

11. Pirri C, Katris P, Trotter J et al (2011) Risk factors at pretreatment predicting treatment-induced nausea and vomiting in Australian cancer patients: a prospective, longitudinal, observational study. Support Care Cancer 19:1549-1563

12. du Bois A, Meerpohl HG, Vach W et al (1992) Course, patterns, and risk-factors for chemotherapyinduced emesis in cisplatin-pretreated patients: a study with ondansetron. Eur J Cancer 28:450-457

13. Hayashi T, Shimokawa M, Matsuo K et al (2018) Risk factors for delayed chemotherapy-induced nausea and vomiting with low-emetic-risk chemotherapy: a prospective, observational, multicenter study. Cancer Manag Res 10:4249-4255

14. lihara $\mathrm{H}$, Shimokawa $\mathrm{M}$, Hayashi T et al. A Nationwide, Multicenter Registry Study of Antiemesis for Carboplatin-Based Chemotherapy-Induced Nausea and Vomiting in Japan. The Oncologist 2019; 25

15. Yoshida I, Tamura K, Miyamoto T et al (2019) Prophylactic Antiemetics for Haematological Malignancies: Prospective Nationwide Survey Subset Analysis in Japan. In Vivo 33:1355-1362

16. Grunberg SM, Deuson RR, Mavros P et al (2004) Incidence of chemotherapy-induced nausea and emesis after modern antiemetics. Cancer 100:2261-2268

17. Molassiotis A, Saunders MP, Valle J et al (2008) A prospective observational study of chemotherapyrelated nausea and vomiting in routine practice in a UK cancer centre. Support Care Cancer 16:201208

18. Ng TL, Hutton B, Clemons M (2015) Chemotherapy-Induced Nausea and Vomiting: Time for More Emphasis on Nausea? Oncologist 20:576-583

19. Warr DGHP, Gralla RJ et al. Efficacy and tolerability of aprepitant for the prevention of chemotherapyinduced nausea and vomiting in patients with breast cancer after moderately emetogenic chemotherapy. J Clin Oncol 2005;23: 2822-2830 [published correction appears in J Clin Oncol 2005;14:5851]. J Clin Oncol 2005;23:2822- 2830

20. Glaus A, Knipping C, Morant R et al (2004) Chemotherapy-induced nausea and vomiting in routine practice: a European perspective. Support Care Cancer 12:708-715

21. Natale JJ (2018) Overview of the prevention and management of CINV. Am J Manag Care 24:S391S397

22. Vidall C, Fernandez-Ortega P, Cortinovis D et al (2015) Impact and management of chemotherapy/radiotherapy-induced nausea and vomiting and the perceptual gap between oncologists/oncology nurses and patients: a cross-sectional multinational survey. Support Care Cancer 23:3297-3305

23. Eisenberg P, Figueroa-Vadillo J, Zamora R et al (2003) Improved prevention of moderately emetogenic chemotherapy-induced nausea and vomiting with palonosetron, a pharmacologically novel 5-HT3 receptor antagonist: results of a phase III, single-dose trial versus dolasetron. Cancer 98:2473-2482 
24. Gralla R, Lichinitser M, Van Der Vegt S et al (2003) Palonosetron improves prevention of chemotherapy-induced nausea and vomiting following moderately emetogenic chemotherapy: results of a double-blind randomized phase III trial comparing single doses of palonosetron with ondansetron. Ann Oncol 14:1570-1577

25. Poli-Bigelli S, Rodrigues-Pereira J, Carides AD et al (2003) Addition of the neurokinin 1 receptor antagonist aprepitant to standard antiemetic therapy improves control of chemotherapy-induced nausea and vomiting. Results from a randomized, double-blind, placebo-controlled trial in Latin America. Cancer 97:3090-3098

26. Hesketh PJ, Grunberg SM, Gralla RJ et al (2003) The oral neurokinin-1 antagonist aprepitant for the prevention of chemotherapy-induced nausea and vomiting: a multinational, randomized, doubleblind, placebo-controlled trial in patients receiving high-dose cisplatin-the Aprepitant Protocol 052 Study Group. J Clin Oncol 21:4112-4119

\section{Figures}




\section{Incidence of nausea}

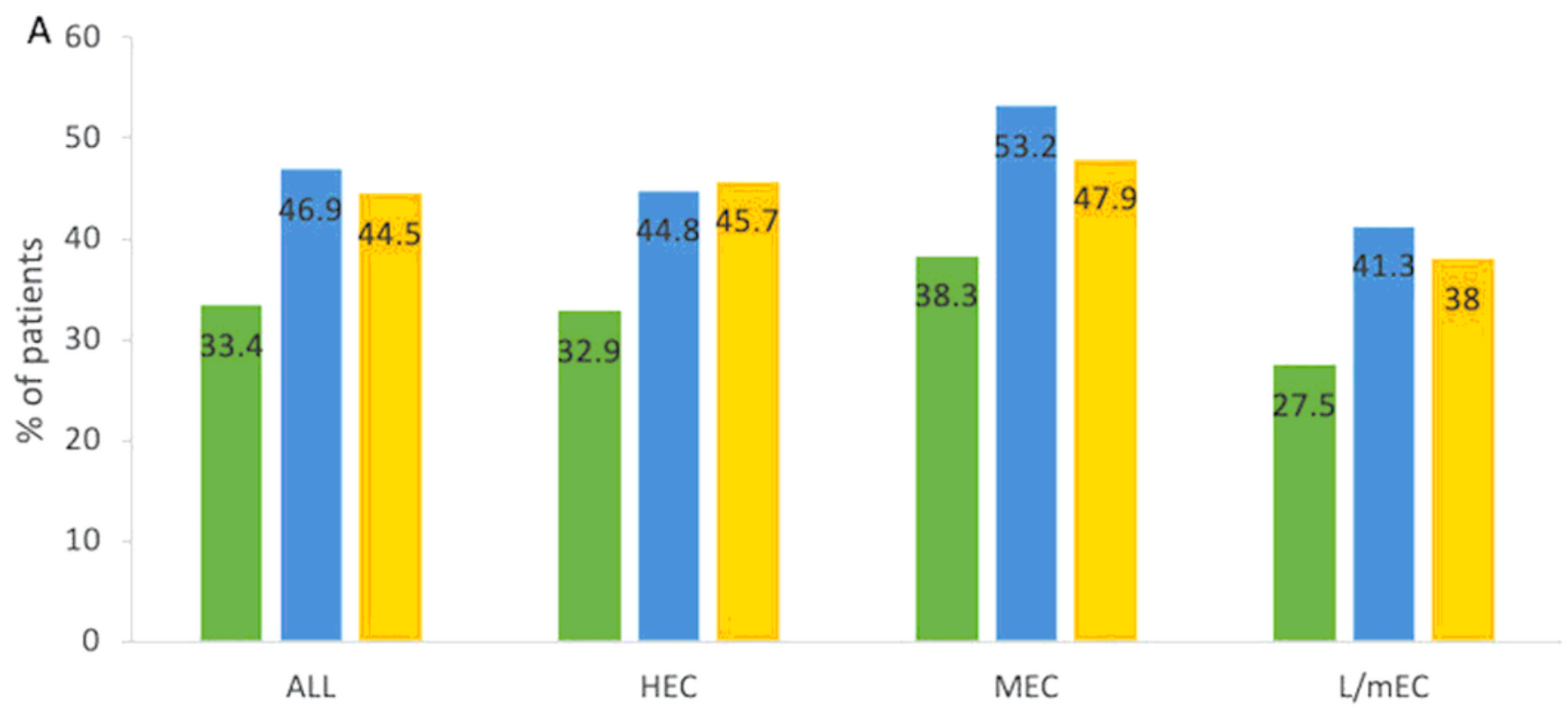

Incidence of vomiting

B 60

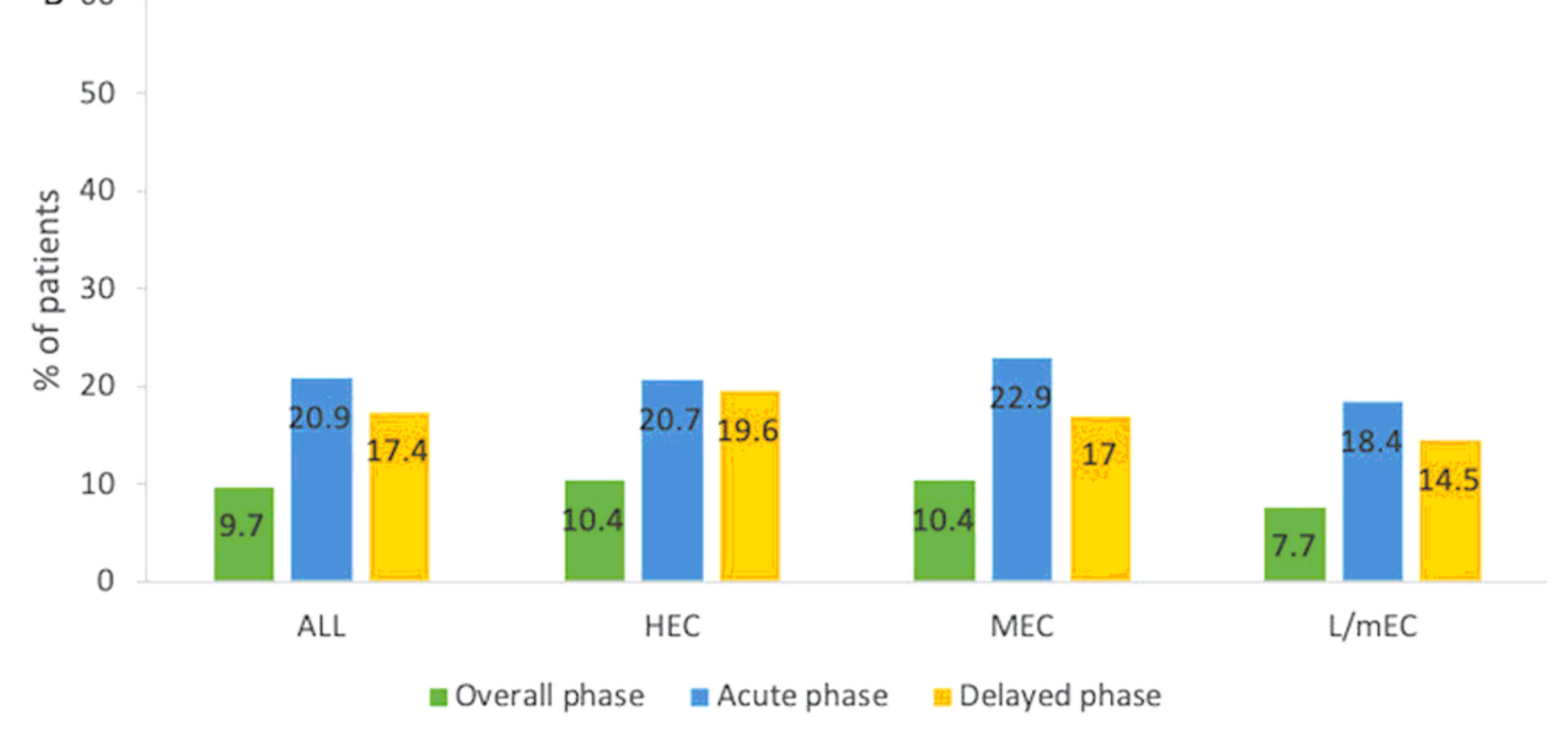

\section{Figure 1}

Incidence of chemotherapy-induced nausea and vomiting among patients who received HEC (highly emetogenic chemotherapy), MEC (moderately emetogenic chemotherapy) , L/mEC (low/minimally emetogenic chemotherapy) and all patients during acute, delayed and overall phases. (A): Incidence of nausea. (B): Incidence of vomiting. Abbreviation: All, the whole study population; HEC, highly emetogenic 
chemotherapy $\mathbb{M E C}$, moderately emetogenic chemotherapy; L/MEC, low/minimally emetogenic chemotherapy.

Overall CR
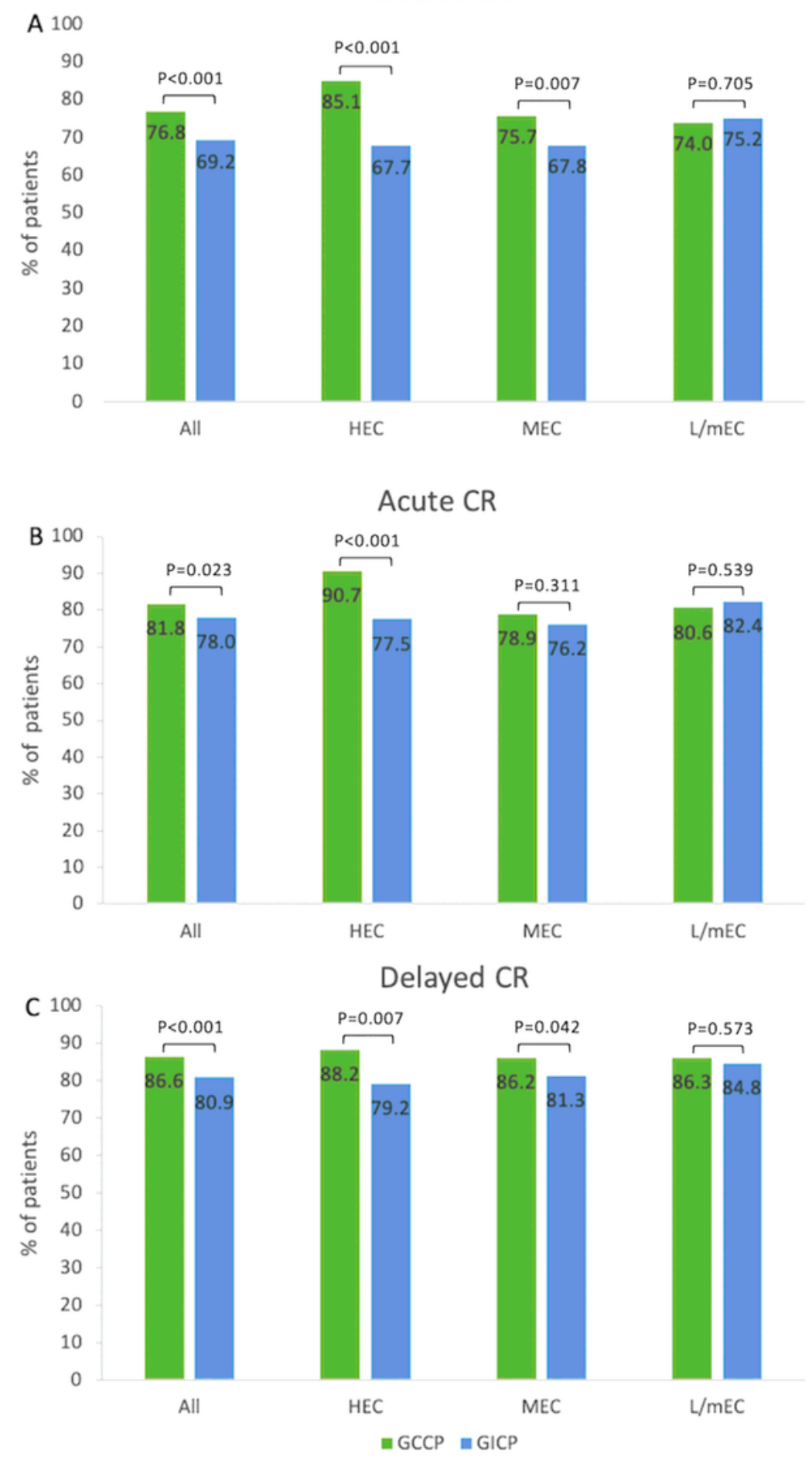

Figure 2

Incidence of CR compared between GCCP and GICP during acute, delayed and overall phases after the most recent chemotherapy. (A): The graph shows the Incidence of overall CR between GCCP and GICP in patients receiving HEC, MEC and L/mEC, respectively, and in the whole study population. (B) The graph 
shows the Incidence of acute CR between GCCP and GICP in patients receiving HEC, MEC and L/mEC, respectively, and in the whole study population. (C) The graph shows the Incidence of delayed CR between GCCP and GICP in patients receiving HEC, MEC and L/mEC, respectively, and in the whole study population. The data are presented as percentages and were tested using the chi-square test.

Abbreviation: : All, the whole study population; HEC, highly emetogenic chemotherapy $\triangle$ MEC, moderately emetogenic chemotherapy; L/MEC, low/minimally emetogenic chemotherapy; GCCP『guideline-consistent

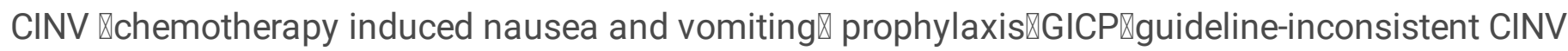
\chemotherapy induced nausea and vomiting $₫$ prophylaxis (GCCP/GICP) $₫ C R \rrbracket$ complete response.

\section{Supplementary Files}

This is a list of supplementary files associated with this preprint. Click to download.

- Supplemental1.docx

- Supplementaltables.docx 